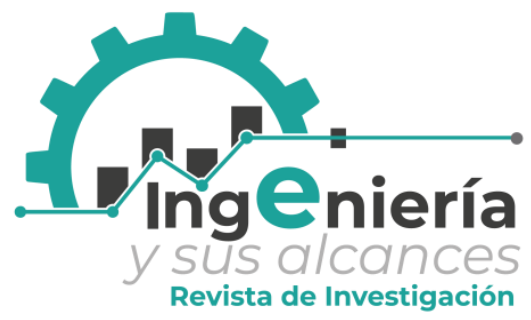

Ingeniería y sus alcances, Revista de Investigación Https://doi.org/10.33996/revistaingenieria.v4i10.65

Septiembre - diciembre 2020

Volumen 4 / No. 10

ISSN: $2664-8245$

ISSN-L: $2664-8245$

pp. 178 - 191

\title{
Análisis hidrológico e hidráulico del potencial de inundación
}

\author{
Hydrological and hydraulic analysis of flood potential
}

Análise hidrológica e hidráulica do potencial de inundação

\author{
Mario Andrés Bladés Benítez \\ marioblades@gmail.com \\ ORCID 0000-0003-0019-7483
}

Juan Gabriel López Soto
gabriel0001020@gmail.com
ORCID 0000-0003-3262-8081

Universidad Católica Boliviana San Pablo, Bolivia

Artículo recibido mayo 2020 | Arbitrado en julio 2020 | Publicado en septiembre 2020

RESUMEN

La cuidad de Tarija - Bolivia enfrenta permanentes inundaciones en zonas urbanas producto de su crecimiento y desarrollo hacia las zonas naturales. Esta investigación se propuso realizar modelaciones hidrológicas e hidráulicas de las subcuencas de la quebrada El Monte que permitan determinar la vulnerabilidad de los terrenos y obras civiles próximas; mediante un estudio descriptivo de corte transversal y enfoque cuantitativo en el que se realizaron evaluaciones hidrológicas e hidráulicas a través de los programas HEC-HMS y HEC-RAS. Como resultado se determinaron zonas con mayor peligro de inundación: las zonas Cine la Torre y Tarija Tenis Club se afectan en un periodo de retorno de 10 años; la zona Hospital Obrero, en 25 años; y la zona Coliseo Universitario, en 50 años. Se concluyó la urgencia de tomar medidas que implican reordenamiento territorial de la ciudad y desarrollo de obras civiles, para prevenir daños significativos en la población en los próximos 10 años.

Palabras clave: Hidrológico; Hidráulico; Inundación; Crecimiento Territorial; Modelación

\section{ABSTRACT}

Tarija - Bolivia faces permanent floods in urban areas because of its growth and development towards natural areas. This research was proposed to carry out hydrological and hydraulic modeling of the sub-basins of the El Monte creek to determine the vulnerability of the nearby lands and civil works; through a descriptive cross-sectional study and quantitative approach in which hydrological and hydraulic evaluations were carried out through the HEC-HMS and HEC-RAS programs. As a result, areas with the greatest risk of flooding were determined: Cine la Torre and Tarija Tenis Club areas are affected in a return period of 10 years; Hospital Obrero, in 25 years; and University Coliseum area, in 50 years. The urgency of taking measures that involve the territorial reorganization of the city and development of civil works was concluded, to prevent significant damage to the population in the next 10 years.

Key words: Hydrological; Hydraulic; Flood; Territorial Growth; Modeling

\section{RESUMO}

A cidade de Tarija - Bolívia enfrenta inundações permanentes em áreas urbanas como resultado de seu crescimento e desenvolvimento em áreas naturais. Esta investigação foi proposta para realizar modelagem hidrológica e hidráulica das sub-bacias do riacho El Monte para determinar a vulnerabilidade das terras próximas e obras civis; por meio de um estudo transversal descritivo e de abordagem quantitativa em que foram realizadas avaliações hidrológicas e hidráulicas por meio dos programas HECHMS e HEC-RAS. Como resultado, foram determinadas as áreas de maior risco de inundação: as áreas do Cine la Torre e Tarija Tenis Club são afetadas em um período de retorno de 10 anos; a área do Hospital Obrero, em 25 anos; e a área do Coliseu Universitário, em 50 anos. Concluiu-se a urgência de tomar medidas que envolvam a reorganização territorial da cidade e o desenvolvimento de obras civis, de forma a evitar danos significativos à população nos próximos 10 anos.

Palavras-chave: Hidrológico; Hidráulico; Inundar; Crescimento Territorial; Modelagem 


\section{INTRODUCCIÓN}

Las crecidas extremas son un fenómeno hidrológico que se caracterizan por ocasionar daños debido a la variación extrema de caudales, poniendo en riesgo vidas humanas y pérdidas materiales. En Bolivia, el crecimiento territorial no planificado incrementa el impacto de desastres naturales (Ministerio de desarrollo Rural, 2008).

El río Guadalquivir es un río del sur de Bolivia que atraviesa la provincia de Cercado, específicamente Tarija, la ciudad capital; se divide en subcuencas que han sido objeto de diferentes estudios de índole ambiental. Esta cuidad se ha visto afectada notablemente por el crecimiento urbano; la expansión espacial se ha desarrollado de forma desordenada y poco planificada, impulsada principalmente por el crecimiento demográfico. Las superficies de los barrios de La Pampa, Virgen de Fátima, El Tejar, Aniceto Arce, Juan XXIII y San Gerónimo, han crecido muy próximas a la quebrada El Monte, en los últimos años casi han duplicado sus áreas construidas y han incluido el cambio substancial de los usos y coberturas de los suelos.

Superficies de suelos con coberturas naturales como bosques, lechos de ríos y quebradas han sido urbanizadas, perturbando severamente los flujos de materia y energía al interior de las cuencas donde se localizan estos barrios. En particular el uso de la tierra y el cambio de gestión de la tierra afectan a la hidrología disminuyendo los recursos hídricos, incrementando el riesgo de inundación.

Las quebradas con más presencia urbana en la provincia de Cercado son El Monte y San Pedro. Históricamente esta provincia ha sido afectada en diversas ocasiones por desastres naturales causados por el rio Guadalquivir y su afluente; resulta necesario realizar un estudio para determinar las zonas con mayor riesgo, especialmente por inundación. Esta investigación se propuso realizar modelaciones hidrológicas e hidráulicas de las subcuencas hidrográficas de la quebrada El Monte que permitan determinar la vulnerabilidad de los terrenos y obras civiles próximas.

La investigación se justifica porque llena un vacío de información, responde al problema de escasez de información de zonas de inundación. Esta es una de las razones por las que existe un mal ordenamiento territorial. La investigación dio a conocer la situación de riesgo de algunas zonas y construcciones: Hospital Obrero, Zona de restaurantes Avenida Los Membrillos, Universidad Autónoma Juan Misael Saracho y Club tenis Tarija. Las simulaciones de inundación permitieron visualizar señales de alerta a consecuencias de crecidas de la quebrada El Monte, demostrando que existen zonas vulnerables que corren peligro de inundación, exponiendo en riesgo vidas humanas, pérdidas económicas $\mathrm{y}$ materiales.

\section{MÉTODO}

Se trató de un estudio de tipo cuantitativo, corte transversal y alcance descriptivo, que tuvo como fin realizar el análisis hidrológico de la quebrada El Monte. Se tomaron como referencia preliminar estudios relacionados: control de inundaciones de la ciudad de Tarija (Sistema Nacional de Cartografía de Zonas Inundables, 1999) (Terrazas, 2011) y (Olaguivel, 1999); estudio de avenidas máximas para prevenir inundaciones (Wayar, 2014). 
El proceso implicó delimitar la cuenca y subcuencas en las cartas topográficas que aporten flujo a la Quebrada El Monte; seleccionar fuente más representativa de datos hidrológicos y aplicar el método de la curva del mundo; determinar las características necesarias de cada subcuenca para la modelación hidrológica; realizar el modelo hidrológico preliminar para diferentes periodos de retorno con el programa HEC-HMS. Los resultados se presentan en dos etapas: estudios hidrológico e hidráulico de la cuenca y análisis de los resultados.

\section{Estudios hidrológico e hidráulico}

Se delimitó la cuenca de la quebrada y sus diferentes subcuencas, haciendo empleo de: las cartas geográficas proporcionadas por el IGM; el Software ArcGIS; y se verificó la exactitud y la similitud entre ambos métodos; optando por el valor resultante de mencionado software, se procedió a medir las longitudes de los ríos principales de cada subcuenca y el río principal hasta la unión con el afluente de la quebrada San Pedro. Se generaron perfiles de los ríos para determinar la pendiente media con la diferencia de alturas que presenta el terreno. El tiempo de concentración se calculó con la fórmula de Kerby y Kirpich. En consecuencia, se calculó el tiempo de retardo. El número de curva fue obtenido por el análisis del tipo de vegetación que existe en la cuenca, el cual fue modelado con software ArcGIS y por consiguiente proporcionó valores característicos de cada subcuenca.
Para la selección de estaciones pluviométricas se tomó en cuenta las que están cerca de la cuenca y que contienen la mayor cantidad de años de registro de precipitaciones máximas en 24 horas. Posteriormente, se aplicó la ley de distribución de Valor Extremo Tipo I, determinando la altura de precipitación para los periodos de retorno de 2, 5, 10, 25, 50, 100, 300 y 500 años. Para determinar alturas menores a 24 horas se recurrió al uso de la curva del mundo que proporciona una tabla que contiene coeficientes que permite obtener alturas de precipitación para tiempos menores a 24 horas.

Se generaron modelos hidrológicos de cada subcuenca utilizando el método de hidrograma unitario, tomando como parámetros: el número de curva, el factor pico, factor de Muskingum y Lag Time. Introduciendo estos valores al software HEC-HMS, se simuló el comportamiento de la cuenca y se generaron caudales pico en cada punto de aforo. Asimismo, se aplicó el programa Arc Gis, utilizando un modelo digital de elevaciones y las cartas geográficas del IGM. para realizar el cálculo del número de curva se utilizó el método tradicional SCS para abstracciones utilizando datos del Proyecto Zonisig, recopilados en 1999-2001 (Proyecto Zonisig, 2002). Las precipitaciones máximas en 24 horas para diferentes periodos de retorno se calcularon mediante la distribución estadística de Valor Extremo Tipo I; sin embargo, por la falta de datos de estaciones pluviométricas y meteorológicas, se aplicó una prueba de bondad de ajuste de Smirnov Kolmogorov. 


\section{Análisis de resultados}

Se presenta un análisis interpretativo de los hallazgos generados por la modelación. Se definieron las áreas afectadas a 5, 10, 25, 50, 100, 300 y 500 años.

Se recopiló una serie de eventos de la historia la ciudad de Tarija relacionados con desastres naturales causados por inundaciones: el registro de precipitación máxima diaria muestra un evento histórico en enero de 1966, una lluvia de 106 mm en la estación Aeropuerto, la cual se encuentra dentro del área urbana (SENAMHI, 2017); en febrero de 1999, Tarija fue declarada zona de desastre natural, debido a una lluvia y la posterior crecida de los ríos y quebradas tanto de la provincia Cercado como de las comunidades vecinas, el fenómeno fue verificado y confirmado a través del modelo realizado para un periodo de retorno de 5 a 10 años en el cual se representa este evento de magnitud con un caudal similar a los 74,7 m3/s (IDEAM, 2014); en el año 2002, en la estación San Andrés del área rural, se registró una lluvia de 150,3 mm, que causó pérdidas económicas y desbordes del río en áreas urbanas (SENAMHI, 2017); año 2000, en la estación de San Jacinto Sud en parte de la subcuenca de San Jacinto se registró una lluvia de 110,5 mm (SENAMHI, 2017); enero de 2008, Tarija se declaró en alerta ante posibles desbordes de ríos y quebradas, evento evaluado para un periodo de retorno de 2 años con un caudal aproximado de 18,3 a 19 m3/s; enero de 2011 (El País, febrero 2015), precipitaciones pluviales provocaron el desborde de las quebradas y cortes intempestivos de energía eléctrica. Este desborde fue validado con la certeza de su ocurrencia en un periodo de retorno de 2 años, donde se tuvo un caudal de $40 \mathrm{~m} 3 / \mathrm{s}$ (El Diario Nacional, febrero 2017) y una precipitación registrada de $85 \mathrm{~mm}$ en la estación Aeropuerto, causando el desborde del río hacia la avenida Felipe Palazón en el barrio de Miraflores (SENAMHI, 2017).

Esta relación histórica denota la necesidad de realizar estudios que permitan determinar las zonas de riesgo, para la posterior toma de decisiones.

\section{Estudios hidrológico e hidráulico}

El estudio hidrológico de la cuenca de la quebrada El Monte requirió delimitar para cada subcuenca: área, longitud, pendientes, velocidad, coeficiente de retraso y tiempo de concentración. Los resultados de estas mediciones se presentan en las tablas 1 y 2 .

Tabla 1. Área y longitud de la cuenca de la quebrada El Monte

\begin{tabular}{cccc}
\hline Subcuencas & Área (Km2) & $\begin{array}{c}\text { Longitud Río } \\
\text { AutoCAD(km) }\end{array}$ & $\begin{array}{c}\text { Longitud Río Google } \\
\text { Earth(km) }\end{array}$ \\
\hline M1 & 9 & 7.447 & 8.19 \\
M2 & 10.88 & 6.291 & 6.63 \\
M3 & 16.05 & 6.365 & 6.97 \\
M4 & 5.54 & 4.704 & 4.59 \\
M5 & 5.35 & 3.681 & 4.43 \\
M6 & 4.05 & 3.565 & 3.72 \\
\hline T0TAL & $\mathbf{5 . 8 8}$ & $\mathbf{1 8 . 3 8}$ & $\mathbf{2 0 . 5}$ \\
\hline
\end{tabular}


En la Tabla 1 se presenta el área de cada subcuenca y la longitud del rio principal, con datos obtenidos mediante el programa AutoCAD Civil 3D y Google Earth.

Tabla 2. Propiedades de la cuenca de la quebrada El Monte

\begin{tabular}{|c|c|c|c|c|c|c|c|c|c|c|}
\hline \multirow[b]{2}{*}{ Subcuenca } & \multicolumn{2}{|c|}{ Pendientes medias } & \multirow{2}{*}{$\begin{array}{c}\text { Pendiente } \\
\text { para } \\
\text { Kerby }\end{array}$} & \multirow{2}{*}{$\begin{array}{c}\text { Coeficientes } \\
\text { de Retraso } \\
\text { de Kerby }\end{array}$} & \multirow{2}{*}{$\begin{array}{c}\text { Velocidad } \\
\mathrm{m} / \mathrm{s}\end{array}$} & \multicolumn{3}{|c|}{ Tiempo total de concentración } & \multicolumn{2}{|c|}{$\begin{array}{l}\text { Factores para } \\
\text { Lag Time }\end{array}$} \\
\hline & $\begin{array}{c}\text { Pendiente } \\
\text { MediaDH/L } \\
\text { (\%) }\end{array}$ & $\begin{array}{l}\text { Pendiente } \\
\text { método de } \\
\text { áreas }\end{array}$ & & & & $\begin{array}{l}\text { Tc Kirpich } \\
\text { (min) }\end{array}$ & $\begin{array}{c}\text { Tc Kerby } \\
\text { (min) }\end{array}$ & $\begin{array}{l}\text { Tc } \\
\text { total }\end{array}$ & Factor & $\begin{array}{l}\text { Lang } \\
\text { time }\end{array}$ \\
\hline M1 & - & 0.0219 & 0.1912 & 0.4 & 1.45 & 87.605 & 6.78 & 94.39 & 0.7 & 66.07 \\
\hline M2 & - & 0.0232 & 0.1897 & 0.4 & 1.39 & 72.726 & 6.792 & 79.52 & 0.7 & 55.66 \\
\hline M3 & - & 0.0202 & 0.0227 & 0.37 & 1.28 & 79.711 & 10.783 & 90.49 & 0.6 & 54.3 \\
\hline M4 & 0.0063 & - & 0.0083 & 0.1 & 0.78 & 90.449 & 7.908 & 98.36 & 0.4 & 39.34 \\
\hline M5 & - & 0.0106 & 0.075 & 0.02 & 1 & 72.09 & 2.085 & 74.18 & 0.4 & 29.67 \\
\hline M6 & 0.0051 & - & 0.0051 & 0.02 & 0.71 & 83.501 & 3.92 & 87.42 & 0.4 & 34.97 \\
\hline
\end{tabular}

Según se observa en la Tabla 2, el tiempo de concentración fue determinado mediante los métodos de Kerby y Kirpich; para el cálculo por Kerby se tomó los primeros 30 metros de la parte superior de la cuenca por las condiciones del terreno de tipo montañoso-escarpado. Asimismo, se aplicó el método de Kirpich siendo esta admisible para el tipo de pendiente y las condiciones de terreno. Por último, para el calculó del tiempo de retardo se aplicó el factor según el SCS, tomando valores entre 0,4 - 0,7 ya que, según la delimitación de las subcuencas, están distribuidas en las zonas rurales y urbanas.

Para el tránsito de hidrogramas se aplicó el método de Muskingum y Lag Time, el cual con lleva la cantidad de segmentos necesarios, la distancia, las cotas y pendientes de cada segmento para poder transportar los hidrogramas de cada subcuenca hacia un mismo punto de unión haciendo una comparación entre los dos métodos y escogiendo el más representativo, se presentan en la Tabla 3.

Tabla 3. Propiedades geométricas para Muskingum

\begin{tabular}{ccccccc}
\hline & \multicolumn{3}{c}{ Propiedades geométricas } & \multicolumn{3}{c}{ Cálculo de pendiente } \\
SEGMENTO & ACAD & $\begin{array}{c}\text { Google } \\
\text { Earth } \\
\mathbf{( k m )}\end{array}$ & $\begin{array}{c}\text { Longitud de } \\
\text { cálculo (m) }\end{array}$ & $\begin{array}{c}\text { Cota } \\
\text { Mayor }\end{array}$ & $\begin{array}{c}\text { Cota } \\
\text { Menor }\end{array}$ & Pendiente \\
\hline EM 1 & 4.330 & 4.330 & 4330 & 1934 & 1898 & 0.0083 \\
EM 2 & 4.704 & 4.590 & 4590 & 1898 & 1869 & 0.0063 \\
EM 3 & 3.565 & 3.720 & 3720 & 1869 & 1850 & 0.0051 \\
\hline
\end{tabular}

En la Tabla 3 se distingue las propiedades geométricas de Muskingum. Nótese que EM2 y EM3 son cauces principales. 
Tabla 4. Resultados Lag time y Muskingum para cada segmento en cuenca El Monte

\begin{tabular}{|c|c|c|c|c|c|c|c|c|c|c|c|}
\hline \multirow[b]{2}{*}{ SEGMENTO } & \multicolumn{4}{|c|}{ Lag Time para segmentos Reach } & \multicolumn{5}{|c|}{ Parámetros Muskingum para cuenca Reachs } & \multicolumn{2}{|c|}{$\begin{array}{c}\text { Cálculo Muskingum } \\
\text { y Lag time }\end{array}$} \\
\hline & $\begin{array}{c}\text { Tc } \\
\text { Kirpich } \\
\text { (min) }\end{array}$ & $\begin{array}{c}\text { Tc } \\
\text { Kerby } \\
\text { (min) } \\
\end{array}$ & $\begin{array}{c}\text { Tc } \\
\text { Total }\end{array}$ & $\begin{array}{c}\text { Lag } \\
\text { time } \\
(\mathrm{min}) \\
\end{array}$ & C & $\begin{array}{c}\text { Muskingum } \\
(\mathrm{x})\end{array}$ & $\min$ & hrs & №Pasos & $\begin{array}{l}\text { Muskingum } \\
\text { (Hr) }\end{array}$ & $\begin{array}{c}\text { Lag } \\
\text { time } \\
(\mathrm{Hr}) \\
\end{array}$ \\
\hline EM 1 & 77.804 & 13.658 & 91.461 & 54.880 & 0.600 & 0.300 & 91.461 & 1.524 & $13-23$ & 1.5243 & 0.9146 \\
\hline EM 2 & 90.449 & 7.908 & 98.357 & 39.340 & 0.600 & 0.300 & 98.357 & 1.639 & $12-27$ & 1.6392 & 0.6557 \\
\hline EM 3 & 83.501 & 3.920 & 87.421 & 34.970 & 0.580 & 0.290 & 87.421 & 1.457 & $11-24$ & 1.4570 & 0.5828 \\
\hline
\end{tabular}

Según se muestra en las dos últimas columnas de la Tabla 4, se realizó una comparación de resultados obtenido mediante el cálculo de Muskingum y Lag time.

Características físicas de la cuenca, el funcionamiento de la cuenca se asemeja al de un colector, que recibe la precipitación pluvial y la convierte en escurrimiento. Esta transformación presenta pérdidas de agua, situación que depende de las condiciones climatológicas y de sus características físicas.

Los parámetros geomorfológicos de la cuenca son: área $A=50.88 \mathrm{~km}^{2}$, perímetro $P$ $=40.27 \mathrm{~km}$, longitud $L=14.34 \mathrm{~km}$, longitud del cauce $L p=10.21 \mathrm{~km}$, cota inicial cauce principal $=10960.39 \mathrm{msnm}$, cota final cause principal $=1883.12 \mathrm{msnm}$, longitud total del cauce $L t=69.58 \mathrm{~km}$, ancho de la cuenca $w$ $=3.55 \mathrm{~km}$, factor de forma de una cuenca $F$ $=0.25$, relación de elongación $R=0.56$, relación de circularidad $R c=0.39$, índice de compacidad $K=1.58$. Asimismo, los parámetros de relieve son: pendiente media de la cuenca $=9.83 \%$, pendiente media del cauce principal $=0.76$. Finalmente, los parámetros de la red hidrográfica son: densidad de drenaje $D d=1.37$, constantes de estabilidad del Río $C=0.73$, densidad de corriente $D c=0.95$.

La cuenca fue dividida en rangos de altitudes para determinar el área comprendida entre estas así usando los valores para crear la curva hipsométrica; para el cálculo de pendiente media de la cuenca se clasifico las pendientes en 7 grupos así tomando el valor de concurrencia de cada uno. Se determinaron 4 cauces. Para el cálculo de numero de curvas (CN) se utilizaron los mapas de suelos, vegetación y usos del suelo del departamento de Tarija. El CN ponderado obtenido fue 78 .

\section{Análisis de precipitaciones máximas} en 24 horas, para realizar el análisis estadístico de los datos pluviométricos se aplicó la distribución de "Valor Extremo tipo I" y Gumbel, según se presenta en la Figura 1, los parámetros de entrada fueron: número de entrada $=73$, media $h d=55.1$, desviación $s d=17.50, \alpha=13.643$, moda $E d=47.27$, característica $K d=0.71$. 


\section{VALOR EXTREMO TIPO}
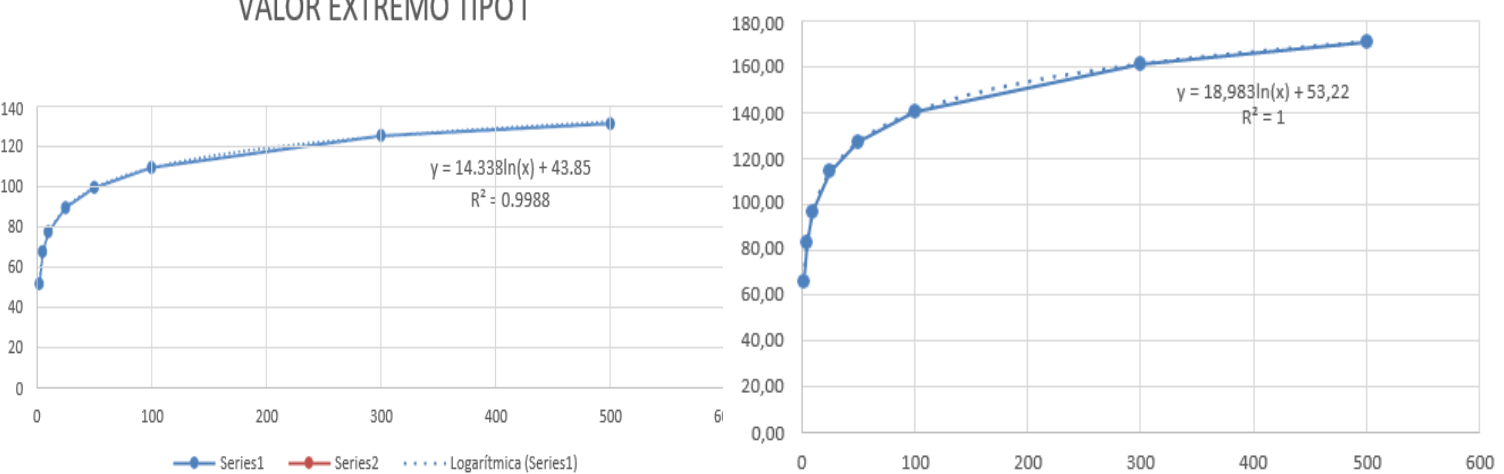

Figura 1. Regresión logarítmica para distribución de "Valor Extremo tipo I" y Gumbel

En la Figura 1 se detalla que el valor de regresión para una distribución de valor extremo tipo I es aproximado a 1 y para la distribución Gumbel es 1, por lo tanto, la distribución es correcta en ambos casos.

Precipitaciones menores a 24 horas, en este caso se consideró la medición de alturas de precipitación para diferentes periodos de retorno, realizada por una investigación previa (UCB, 2017).

Análisis hidrológico HEC-HMS, una vez contó con los parámetros definidos que forman el modelo de la cuenca, las especificaciones de control y estadística de los datos pluviométricos, se procedió a ejecutar la simulación en HEC- HMS. El resultado obtenido son los caudales de punta para cada unión, generados para los periodos de retorno de $2,5,10,25,50,100$, 300 y 500 años. En las figuras 2-7, se muestran los hidrogramas de cada unión para los periodos de retorno de $2,5,10,25$, 50, 100, 300 y 500 años. 

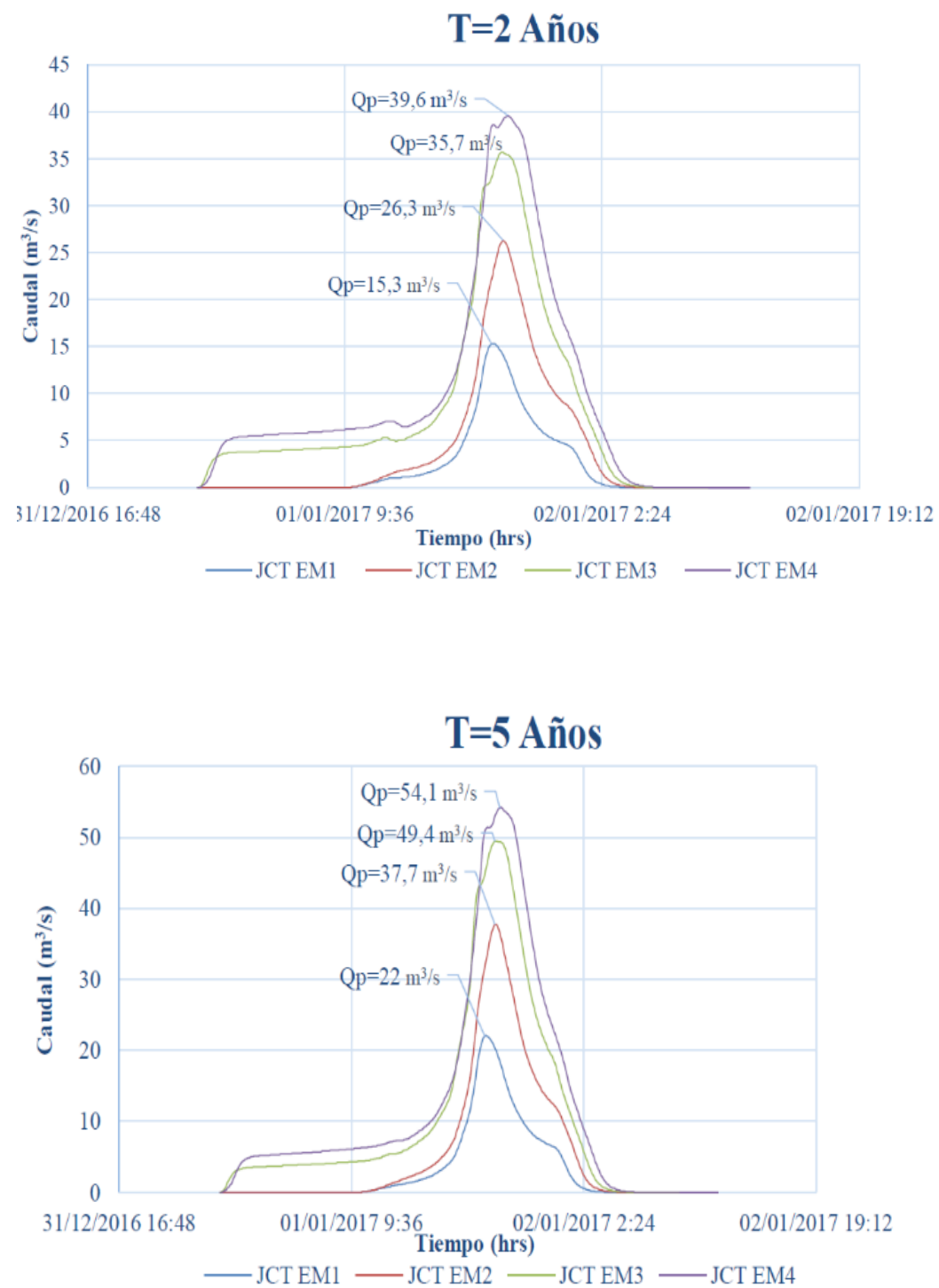

Figura 2. Hidrogramas unitarios para 2 y 5 años

En la parte superior de la Figura 2 se muestra el hidrograma de cada unión, (denominada JCT, por su nombre en inglés Junction), junto al flujo pico de cada una, para un periodo de retorno de 2 años; en la parte inferior, se muestra la misma relación para un periodo de 5 años. 

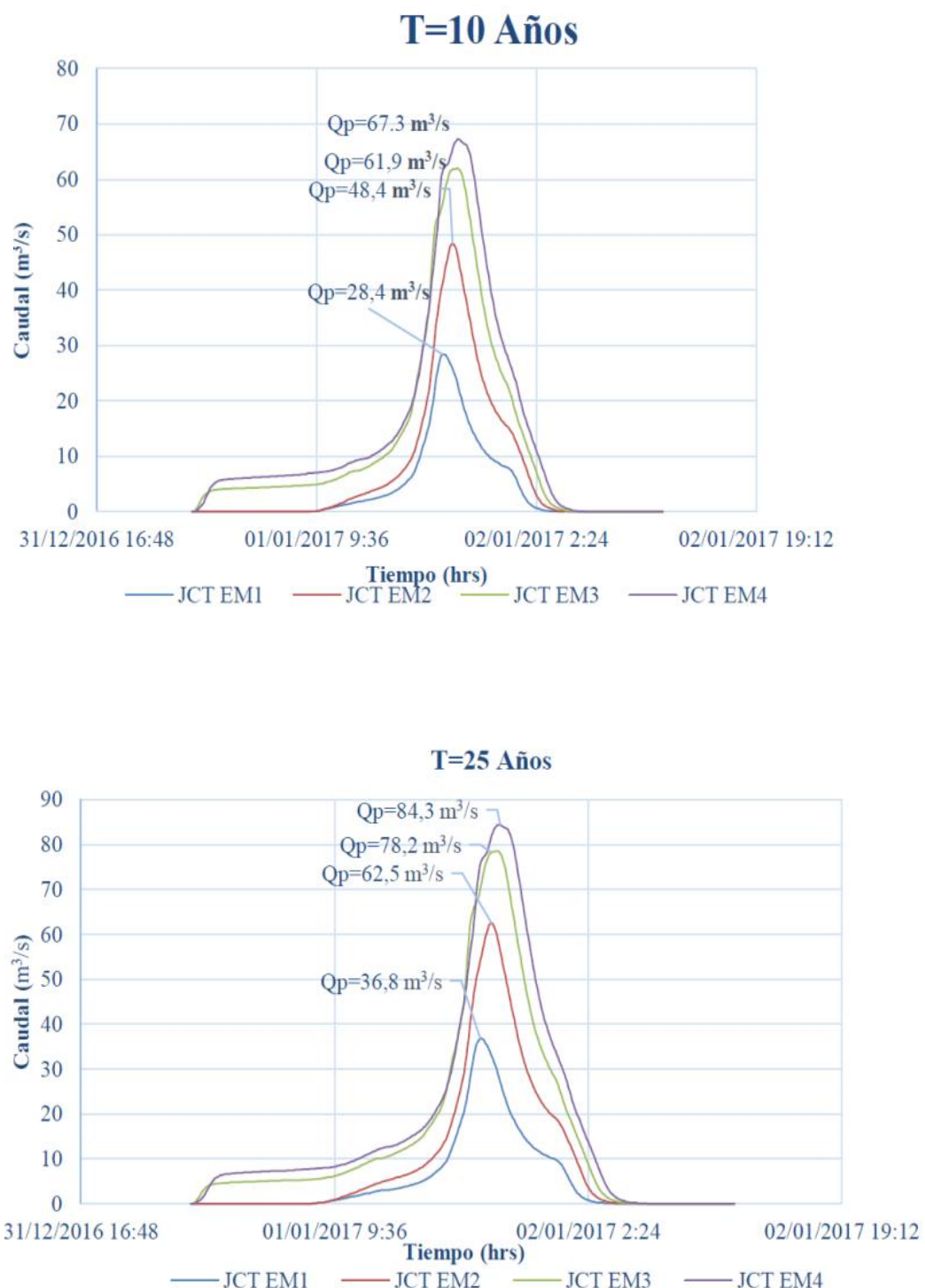

Figura 3. Hidrogramas unitarios para 10 y 25 años

En la parte superior de la Figura 3 se muestra el hidrograma de cada unión y el flujo pico respectivo, para un periodo de retorno de 10 años. En la parte inferior se presenta la misma representación para un periodo de 25 años. 

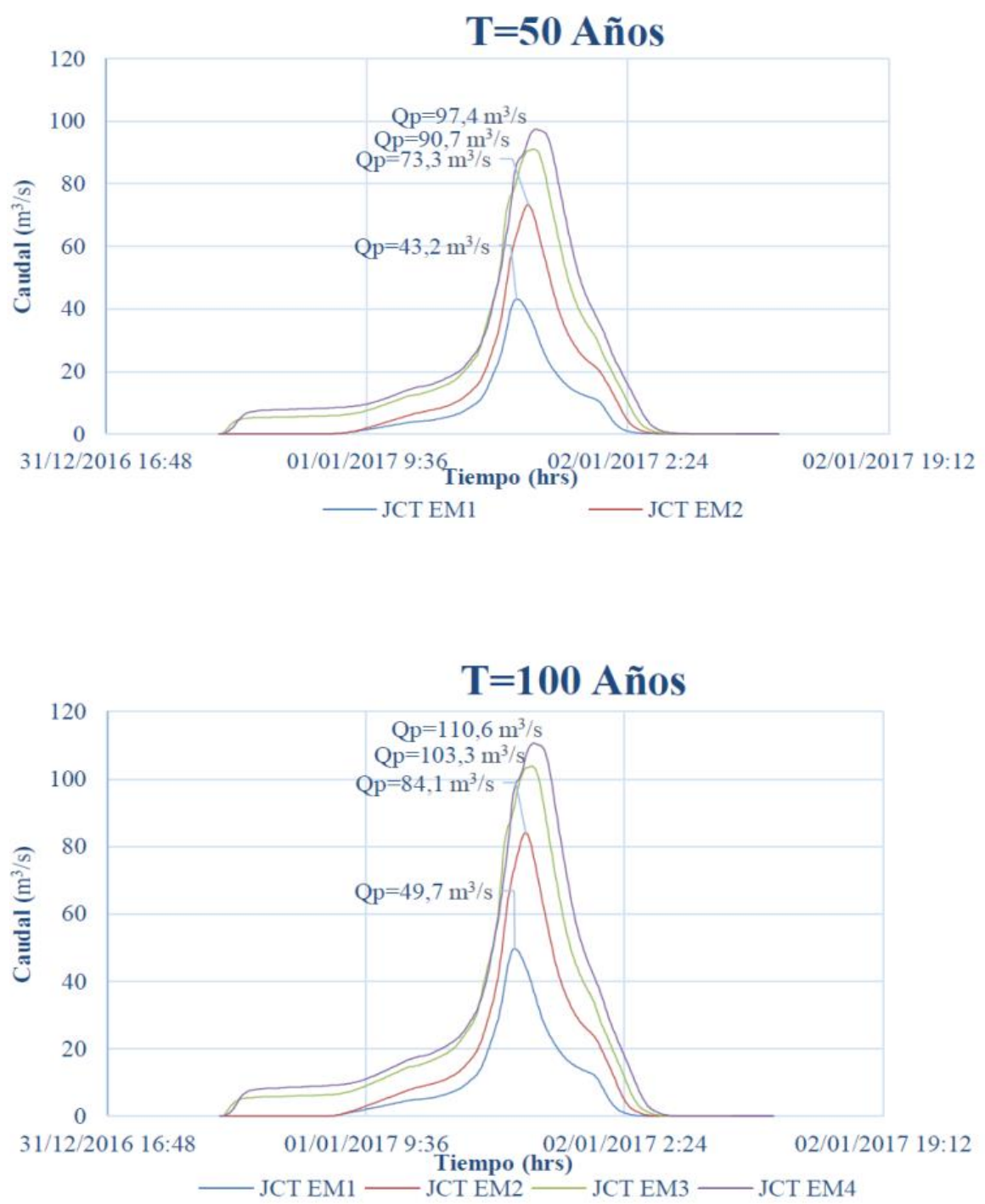

Figura 4. Hidrogramas unitarios para 50 y 100 años

En la parte superior de la Figura 4 se presenta el hidrograma de cada unión y el flujo pico respectivo, para un periodo de retorno de 50 años; en la parte inferior, la misma representación para un periodo de 100 años. 

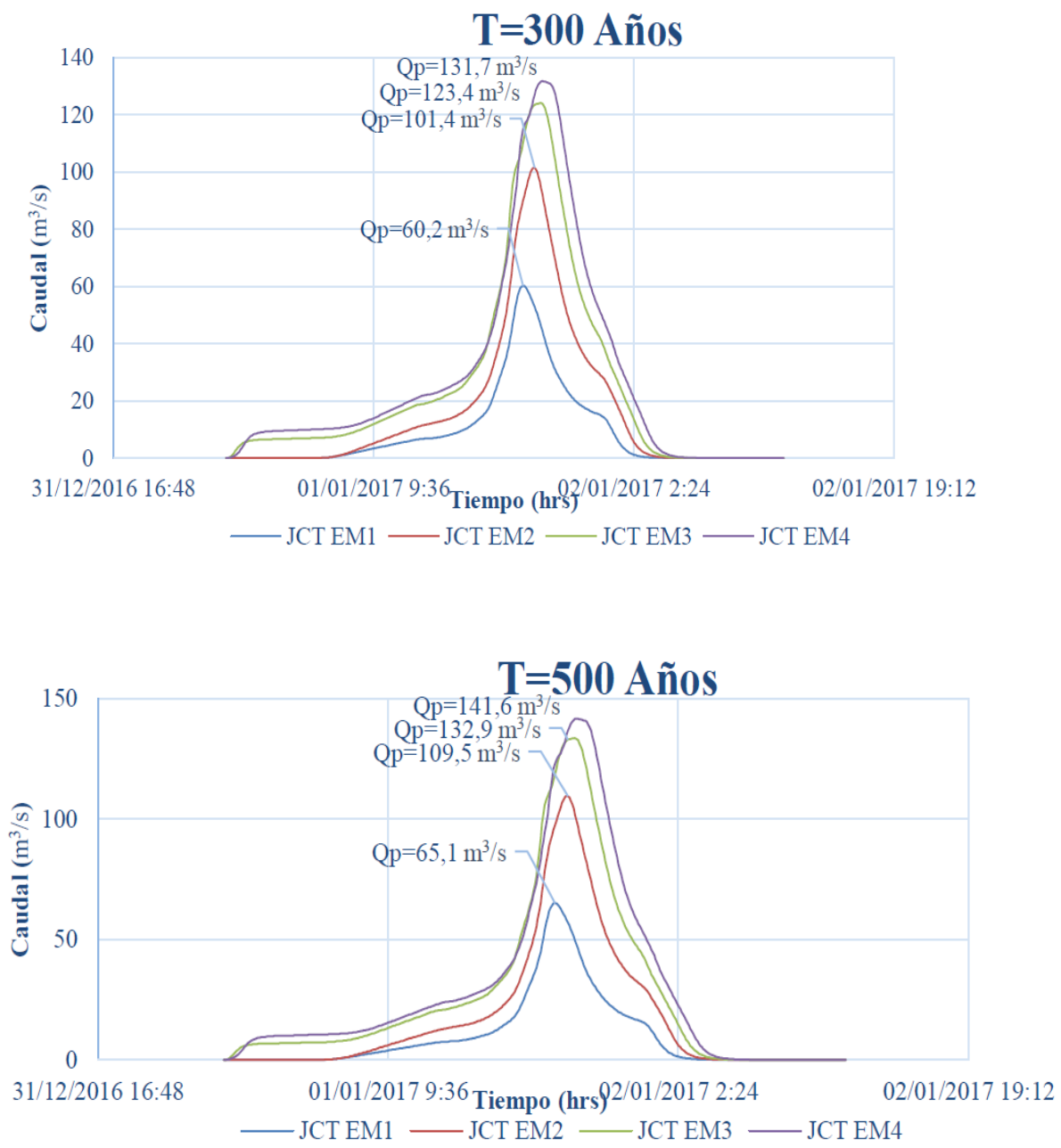

Figura 5. Hidrogramas unitarios para 300 y 500 años

En la parte superior de la Figura 5 se observa el hidrograma de cada unión y el flujo pico respectivo, para un periodo de retorno de 300 años; finalmente, en la parte inferior se presenta la misma representación para un periodo de 500 años.

Los resultados obtenidos se sintetizaron a través del modelaje en el programa HECHMS. Para diferentes periodos de retorno los caudales de pico máximo son: 2 años $=39.6$ $\mathrm{m}^{3} / \mathrm{s} ; 5$ años $=54.2 \mathrm{~m}^{3} / \mathrm{s}, 10$ años $=67.3$ $\mathrm{m}^{3} / \mathrm{s} ; 25$ años $=84.4 \mathrm{~m}^{3} / \mathrm{s}, 50$ años $=97.5$ $\mathrm{m}^{3} / \mathrm{s} ; 100$ años $=110.7 \mathrm{~m}^{3} / \mathrm{s} ; 300$ años $=131.8 \mathrm{~m}^{3} / \mathrm{s}$ y 500 años $=141.7 \mathrm{~m}^{3} / \mathrm{s}$.

\section{Análisis}

La cuenca El Monte está deteriorada, dado que principalmente se encuentra en los valles, presenta pendientes entre $0 \%$ al $12 \%$. $\mathrm{Su}$ forma es alargada y plana, lo que implica que no es muy propensa a tormentas que abarquen toda el área de la cuenca. El hidrograma resultó tener un pico alto y tiempo no prolongado. La corriente de la cuenca es de cuarto orden. 
Los caudales máximos fueron sometidos a pérdidas constantes. En vista de que el uso de suelos varía a lo largo de la cuenca, estudios para los tres factores de pérdidas ayudarían a mejorar los resultados finales. El uso del Proyecto Zonisig para el cálculo del número de curva dio resultados muy próximos a los obtenidos en las diferentes investigaciones ya llevadas a cabo con diferentes métodos. El uso de dos métodos para el tránsito de hidrogramas (Muskingum y Lag Time), toma en cuenta todas las pérdidas mencionadas anteriormente y transporta el hidrograma inicial hacia la confluencia generando una disminución del caudal para la suma final de las subcuencas afluentes a la unión correspondiente.

La zona Hospital Obrero, se ve afectada en los periodos de retorno de 25, 50, 100, 300 y 500 años, específicamente entre las secciones 2789.51 y 2720.91; la zona Cine la Torre se ve afectada en los periodos de retorno de 10, 25, 50, 100, 300 y 500 años, entre las secciones 2451.41 y 2009.21, tiene una zona de baja profundidad debido al puente de la avenida Defensores del Chaco; la zona Coliseo Universitario se ve afectada en los periodos de retorno de 50,100, $300 \mathrm{y}$ 500 años entre las secciones $1939.3 \mathrm{y}$ 1538.84; la zona Tarija Tenis Club se ve afectada en los periodos de retorno de 10 , $25,50,100,300$ y 500 años entre las secciones 720.22 y 287.04, tiene altura de desbordamiento provisional debido a la unión de la Quebrada San Pedro, podría generar un incremento en la mancha de inundación para esta zona.

Los gaviones que existen en la quebrada El Monte en el tramo de estudio son solamente protección de laderas, por lo que resulta insuficiente para el riesgo de inundación existente en la zona.

\section{CONCLUSIONES}

El ordenamiento territorial, es uno de los mayores problemas que enfrenta la ciudad de Tarija, producto de su crecimiento y desarrollo; el crecimiento se ha dado hacia las zonas naturales ocasionando un cambio en el uso de suelo, lo que ha derivado que muchas construcciones se encuentren en las zonas vulnerables a daños provocados por desastres naturales, como es el caso de las inundaciones.

La modelación permitió visualizar señales de alerta, demostrando que existen zonas vulnerables que corren peligro de inundación en periodos de retorno no mayores a 10 años, exponiendo en riesgo vidas humanas, pérdidas económicas y materiales. Se concluyó la urgencia de tomar medidas que implican reordenamiento territorial de la ciudad y desarrollo de obras civiles.

Las precipitaciones calculadas son una aproximación a la realidad por diferentes motivos: limitaciones como datos faltantes, irregularidad en la medida de precipitaciones diarias, de las estaciones, incertidumbre en la topografía y el uso de suelos. La falta de aforos limita la precisión de los resultados, dado que el caudal es cambiante, el tener aforos puede incrementar la exactitud. Los caudales pico para diferentes periodos de retorno calculados mediante el programa HEC-HMS fueron sometidos a diferentes perdidas, por lo tanto, los resultados se constituyen en una base de información que puede ser mejorada en calidad y precisión.

Se resalta la pertinencia y utilidad de realizar comparaciones de diferentes metodologías en el análisis topográfico e hidrográfico de la cuenca en estudio, para obtener los parámetros precisos. Asimismo, 
se debe justificar que el uso del software sea el adecuado para cada caso.

\section{REFERENCIAS}

El Diario Nacional. (7 de febrero de 2017). Obtenido de http://www.eldiario.net/noticias/2017 /2017_02/nt170207/nacional.php?n=3 4

El País. (14 de febrero de 2015). Obtenido de El País online: http://www.elpaisonline.com/index.ph p/2013-01-15-14-1626/sociedad/item/159649-rioguadalquivir-ruge-despues-de-20-anosy-provoca-desborde-peligroso

IDEAM. (2014). Instituto de Hidrología, Meteorología y Estudios Ambientales. Obtenido de http://www.ideam.gov.co/web/atencio n-y-participacion-ciudadana/glosario

Ministerio de Desarrollo Rural. (2008). Mapa de áreas y predios afectados por la inundación provocada por el fenómeno de "la niña 2007 - 2008". La Paz
Olaguivel, Q. C. (1999). Estudio del riesgo de inundación en la quebrada de San Pedro en la ciudad de Tarija

Proyecto Zonisig. (2002). Mapas del departamento de Tarija. CD de uso público, Proyecto Zonisig. (1999-2001)

SENAMHI. (2017). Resumen climatológico. Servicio Nacional de Meteorología e Hidrología, Tarija

Sistema Nacional de Cartografía de Zonas Inundables. (1999). Control de Inundaciones de la Ciudad de Tarija. España

Terrazas, G. A. (2011). Evaluación del riesgo de inundación de la Quebrada el Monte de la ciudad de Tarija. Tarija, Bolivia

UCB. (2017). Investigación del potencial de inundación del Rio Guadalquivir y generación de mapas de inundación. Tarija, Bolivia

Wayar, R. (2014). Estudio de avenidas máximas para prevenir inundaciones del río Pilcomayo en la ciudad de Villa Montes. Tarija, Bolivia 